\title{
Preparo e administração de medicamentos de alta vigilância na perspectiva da
}

\section{segurança do paciente}

\author{
High-surveillance drugs preparation and administration from the perspective of patienty safety \\ Preparación y administración de medicamentos de alta vigilância la perspectiva de seguridade del \\ paciente
}

Recebido: 07/01/2022 | Revisado: 12/01/2022 |Aceito: 15/01/2022 | Publicado: 17/01/2022

\author{
Wylma Danuzza Guimarães Bastos \\ ORCID: https://orcid.org/0000-0002-9908-4237 \\ Universidade Federal de Pernambuco, Brasil \\ E-mail: wylmabastos@yahoo.com.br \\ Priscila Santos Leal \\ ORCID: https://orcid.org/0000-0002-8804-386X \\ Universidade Federal de Pernambuco, Brasil \\ E-mail: priscilasantoslealmoura@gmail.com \\ Jamilly Fernanda Brito Rodrigues \\ ORCID: https://orcid.org/0000-0002-6355-7397 \\ Universidade Federal de Pernambuco, Brasil \\ E-mail: jamillyfernanda_@hotmail.com \\ Dayzyane Farias dos Santos Melo \\ ORCID: https://orcid.org/0000-0001-6095-5691 \\ Universidade Federal de Pernambuco, Brasil \\ E-mail: dayzyane.farias@gmail.com \\ Karina Perrelli Randau \\ ORCID: https://orcid.org/0000-0002-4486-4420 \\ Universidade Federal de Pernambuco, Brasil \\ E-mail: karina.prandau@ufpe.br
}

\begin{abstract}
Resumo
O preparo e administração de medicamentos são etapas no sistema de medicação, sendo a última parte do processo que antecede ao paciente. Um sistema composto por várias entradas e processos e com a participação dos profissionais do nível médio e superior, médicos, enfermeiros, farmacêuticos e técnicos em farmácia e enfermagem. Dentre a responsabilidade medicamentosa, existe um grupo denominado de Medicamentos de Alta Vigilância (MAV) ou Medicamentos Potencialmente Perigosos (MPP), que através da farmacovigilância exigem cuidados mais rigorosos, devido a sua composição e a capacidade de morte que possui, quando administrados de forma errônea. Portanto, esse trabalho tem como objetivo demonstrar a importância do preparo e administração dos MAV/MPP e sua interface com a segurança do paciente. Foi realizada uma revisão narrativa, a partir da pesquisa nas bases de dados eletrônicas Literatura Latino-Americana em Ciências da Saúde (LILACS), BIREME e Scientific Electronic Library Online (SciELO), com o recorte temporal de 2010 a 2021. Após aplicação dos critérios de inclusão e exclusão, e leitura completa dos textos, foram selecionados 10 trabalhos. O levantamento demonstrou a repetição dos erros com MAV envolvendo os medicamentos: heparina, fentanil, midazolam, nalbufina, pancurônio, dopamina, cloreto de potássio, tramadol, epinefrina, petidina, morfina e também com anestésicos e eletrólitos. A pesquisa concluiu sobre a fragilidade dos dados registrados nos artigos encontrados, não categorizando riscos em populações maiores, sendo incipientes e inexpressivos para uma realidade da abrangência do país e/ou por regionalidade.
\end{abstract}

Palavras-chave: Erros de medicação; Lista de medicamentos perigosos; Segurança do paciente.

\begin{abstract}
The preparation and administration of medications are steps in the medication system, being the last part of the process that precedes the patient. A system composed of several inputs and processes and with the participation of mid-level and higher education professionals, physicians, nurses, pharmacists and pharmacy and nursing technicians. Among the drug liability, there is a group called High-Surveillance Drugs or Potentially Dangerous Medicines, so through pharmacovigilance require more rigorous care, due to their composition and the capacity for death they have, when administered in a way erroneous. Therefore, this work aims to demonstrate the importance of the preparation and administration of type medications and its interface with patient safety. A narrative review was carried out based on a search in the Latin American Literature in Health Sciences (LILACS), BIREME and Scientific Electronic Library Online (SciELO) electronic databases, with a time frame from 2010 to 2021. After applying the criteria of
\end{abstract}


inclusion and exclusion, and complete reading of the texts, 10 works were selected. The survey demonstrated the repetition of errors with AVM involving medications: heparin, fentanyl, midazolam, nalbuphine, pancuronium, dopamine, potassium chloride, tramadol, epinephrine, pethidine, morphine and also with anesthetics and electrolytes. The research demonstrated the fragility of the data recorded in the articles found, not categorizing risks in larger populations, being incipient and insignificant for a reality of the scope of the country and/or by regionality.

Keywords: Medication errors; Potentially inappropriate medication list; Patient safety.

\section{Resumen}

La preparación y administración de medicamentos son pasos en el sistema de medicación, siendo la última parte del proceso que precede al paciente. Un sistema compuesto por varios insumos y procesos y con la participación de profesionales de nivel medio y superior, médicos, enfermeras, farmacéuticos y técnicos de farmacia y enfermería. Dentro de la responsabilidad de los medicamentos, se encuentra un grupo denominado Medicamentos de Alta Vigilancia (MAV) o Medicamentos Potencialmente Peligrosos (MPP), que por medio de la farmacovigilancia requieren un cuidado más estricto, por su composición y la capacidad de muerte que tienen, cuando se administran de forma adecuada. Por lo tanto, este trabajo tiene como objetivo demostrar la importancia de la preparación y administración de AVM / MPP y su interfaz con la seguridad del paciente. Se realizó una revisión narrativa a partir de una búsqueda en las bases de datos electrónicas de Literatura Latinoamericana en Ciencias de la Salud (LILACS), BIREME y Scientific Electronic Library Online (SciELO), con un marco temporal de 2010 a 2021. Luego de aplicar los criterios de inclusión y exclusión, y lectura completa de los textos, se seleccionaron 10 obras. La encuesta demostró la repetición de errores con MAV que involucran medicamentos: heparina, fentanilo, midazolam, nalbufina, pancuronio, dopamina, cloruro de potasio, tramadol, epinefrina, petidina, morfina y también con anestésicos y electrolitos. La investigación demostró la fragilidad de los datos registrados en los artículos encontrados, no categorizando riesgos en poblaciones mayores, siendo incipientes e insignificantes para una realidad del ámbito del país y por regionalidad.

Palabras clave: Errores de medicación; Lista de medicamentos potencialmente inapropiados; Seguridad del paciente.

\section{Introdução}

Os incidentes ameaçadores a segurança do paciente continua sendo uma realidade crescente, mesmo com o incremento de novas tecnologias, a susceptibilidade de um evento adverso ainda é inerente ao risco de uma prática assistencial na saúde. O desafio de melhoria na qualidade na saúde é mundial, impulsos terapêuticos e égides sanitárias mobilizam as organizações de saúde a constantes evoluções, como resultado, melhores indicadores e ampliação de processos para promoção da cultura de segurança (Brasil, 2013).

Na mobilização internacional em prol ao movimento de uma prática assistencial segura, Romero et al. (2018) menciona o início deste contexto ético com a publicação de vários relatos após o relatório do Instituto de Medicina (Institute of Medicine - IOM), dos Estados Unidos da América (EUA), em 1999, "Errar é Humano - Construindo um Sistema de Saúde mais Seguro", ficou mais evidente a propagação do conceito do evento adverso como incidente que alcançou o paciente e resultando num dano ou lesão, podendo evoluir para um prejuízo temporário ou permanente.

Neste contexto, o Brasil detectou uma necessidade imperiosa de adequação para gerir os riscos na assistência à saúde em 2002, sendo unificadas instituições de saúde compondo um programa chamado Rede Sentinela, que existe até os dias atuais trabalhando com o gerenciamento de risco sobre os pilares da busca ativa de eventos adversos e uso racional das tecnologias em saúde. Entre os eixos de prioridades, o da farmovigilância vem destacando-se, através do uso racional de medicamentos (Brasil, 2014).

Baseado nesta problemática de gestão de processos envolvidos, a Organização Mundial de Saúde (OMS) lançou a Aliança Mundial para Segurança do Paciente e juntamente com a Comissão Conjunta Internacional (Joint Comission International), adotou as Metas Internacionais de Segurança do Paciente (MISP), constituídas de medidas preventivas nas situações de risco a erros de identificação do paciente, falhas na comunicação, erros de medicação, erros em procedimentos cirúrgicos, infecções associadas à assistência e quedas dos pacientes (Brasil, 2013).

A OMS lançou um novo desafio que envolve a segurança dos pacientes envolvendo medicamentos, sendo intitulada

"Medicações sem danos" (Sheikh et al., 2017). No propósito do controle racional de medicamentos é preocupante visto que 
cerca de 30\% dos danos durante o internamento estão correlacionados a erros de medicamentos (Miasso et al., 2006). Os resultados de quaisquer erros geram consequências que culminam em pontos negativos tanto para os usuários da saúde, e principalmente se for um erro evitável como é um erro relacionado a medicamentos tanto no âmbito individual, como na coletividade, aumentando o tempo do internamento hospitalar, aumentando o risco de infecções, tornando-se mais oneroso e diminuindo a credibilidade na instituição (Curran et al., 2011).

Através de estratégias para controle da situação brasileira, o Ministério da Saúde instituiu o Programa Nacional de Segurança do Paciente (PNSP) em 2013 a todos estabelecimentos de saúde do território nacional (público, privado, filantrópicos) e posteriormente a Agência Nacional de Vigilância Sanitária (ANVISA) criou uma Resolução de Diretoria Colegiada (RDC) $n^{\circ} 36$ de 25 de julho de 2013, contemplando as boas práticas no âmbito hospitalar, instituindo ações em promoção da segurança do paciente e a melhoria da qualidade nos serviços de saúde e a criação do Núcleo de Segurança do Paciente (NSP).

A técnica de medicar um paciente/usuário/cliente, consiste em analisar a prescrição, preparar e administrar o medicamento descrito pelo profissional prescritor, na qual também prescreve a via de administração, sendo que este ato não é tão simples como aparenta, esta etapa está inclusa num amplo e sistemático processo de medicação. Devido esta amplitude dos processos, com várias etapas e profissionais envolvidos antes do preparo e administração, não é detectável a amplitude da multicausalidade que pode acarretar para etapa final com êxito ou não. O erro de medicação é exarado na sua definição como: falhas no processo do tratamento medicamentoso que podem conduzir, ou que tem o potencial para conduzir danos ao paciente (World Health Organization [WHO], 2009).

É válida a avaliação dos fatores de multicausais deste fenômeno na saúde, principalmente o que se refere aos estruturais e humanos, os quais estão diretamente relacionados à assistência à saúde e com a segurança do paciente. No processo avaliativo é possível a identificação em tempo hábil de erros ou near misses (quase-erros), diminuindo as consequências dos agravos, investindo numa cultura de segurança e evitar a recorrência, fortalecendo um sistema eficaz na administração de medicamentos (Brasil, 2013).

E no contexto dos medicamentos potencialmente perigosos, ou seja, medicamentos de alta vigilância (MAV), que possuem um alto risco de provocar danos significativos quando há falha no processo de medicação. Estes erros com MAV não ocorrem frequentemente, porém, quando ocorrem são fatais ou com danos permanentes (Institute For Safe Medication Practices, 2018).

A fragilidade sobre o controle e uso correto de medicamentos torna-se um problema em saúde pública, podendo gerar erros, onerosidade ao sistema de saúde, inviabilidade terapêutica e até a falha, aumentando o número de eventos adversos evitáveis nos pacientes e até a morte. Neste contexto, o objetivo deste artigo é analisar os fatores de multicausalidade no ambiente hospitalar que favorecem ou interferem numa prática segura no processo de preparo e administração de medicamentos de alta vigilância a luz dos critérios de segurança e sanitários exigidos, descrevendo pontos importantes nas etapas do sistema de medicamentos, contribuindo para uma prática assistencial medicamentosa segura ao paciente e aos profissionais envolvidos.

\section{Metodologia}

Trata-se de um estudo de revisão da literatura do tipo narrativa, sendo utilizada uma busca de temática específica, reconhecendo a unidade e a diversidade na interpretação do segmento e desenvolve o conhecimento do conteúdo estudado, através da formulação de uma pergunta norteadora. Este tipo de estudo colabora com a coerência nas argumentações do pesquisador (Echer, 2001). A pergunta da pesquisa foi: quais são os principais entraves a segurança do paciente no preparo e administração de medicamentos de alta vigilância? 
Após esta etapa, a identificação e seleção crítica dos manuscritos científicos foram encontradas nas bases de dados eletrônicas Literatura Latino-Americana em Ciências da Saúde (LILACS), BIREME e Scientific Electronic Library Online (SciELO). A busca de artigos foi realizada por meio das palavras-chaves selecionadas segundo a classificação dos Descritores em Ciências da Saúde (DeCS): Erros de medicação e Erros com medicamentos potencialmente perigosos/Medication Errors, Lista de medicamentos perigosos/Potentially Inappropriate Medication List e Segurança do Paciente/Patient Safety, sendo utilizado na seleção o boleano "AND”. A Legislação vigente sanitária brasileira através da ANVISA e as recomendações da OMS também foram consideradas no estudo.

Para seleção dos artigos realizou-se, primeiramente, a leitura dos resumos das publicações selecionadas com o objetivo de refinar a amostra por meio de critérios de inclusão e exclusão. Os critérios de inclusão foram artigos originais publicados entre 2011 e 2021 e oriundos de estudos desenvolvidos no Brasil. Os critérios de exclusão foram: artigos de revisão ou reflexão, ausência de resumo nas plataformas de busca on-line, artigos repetidos e artigos que não falassem dos medicamentos de alta vigilância.

\section{Resultados}

Por meio do cruzamento com os descritores nas bases de dados, a amostra inicial foi de 111 artigos, sendo descartados 3 artigos duplicados, contendo 108 artigos, após a implantação dos filtros correspondente aos: últimos 10 anos de publicação, idioma em português, e exclusão de artigos de revisão/ e não englobava a temática restaram 10 artigos. A Figura 1 demonstra, através do fluxograma, o método de seleção utilizado para escolha dos estudos.

Figura 1: Fluxograma do método de seleção dos trabalhos.

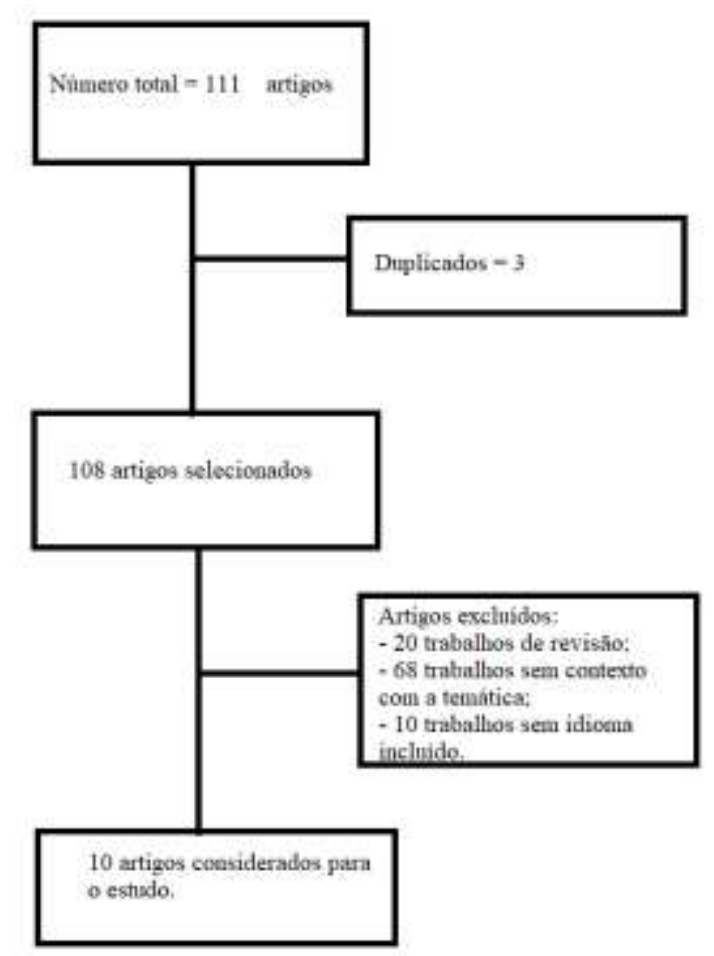

Fonte: Autores (2022). 
Após avaliação crítica na leitura dos artigos selecionados na íntegra, ficou claro que dos 10 artigos, 9 foram pesquisados por enfermeiros e publicados em revista da mesma área, somente 1 artigo foi elaborado por pesquisador farmacêutico e publicado em revista na área das ciências farmacêuticas.

As análises dos artigos revelaram os conceitos e a formação da problematização acerca de uma assistência à saúde com práticas inseguras, na década de 90 quando houve uma movimentação no país para construto da segurança do paciente, através das instituições privadas e públicas, adotando medidas para melhoria da assistência prestada. O Brasil e outros países, tiveram a influência através da mobilização global de ponto de partida da segurança do paciente, através de um relatório publicado nos Estados Unidos com o número de americanos que morriam nos hospitais por eventos adversos, em média de 44.000 e 98.000 mortes por ano e o número de no mínimo 400.000 eventos adversos evitáveis relacionados a medicamentos, e que cada paciente está sujeito a um erro de medicação por dia. Como primeiro passo para medidas profiláticas veio a criação do Programa de Controle da Qualidade Hospitalar (CQH) em 1991(Duarte, et al., 2015; Kohn et al., 2001).

Após a criação do $\mathrm{CQH}$, a temática sobre qualidade em saúde ficou cada vez mais evidente, e consolidou através da acreditação hospitalar, sendo pautada pela primeira vez no Ministério da Saúde em 1995, através do Programa de Garantia e Aprimoramento da Qualidade em Saúde e em 1998 foi lançado o Manual Brasileiro de Acreditação Hospitalar. Em 1999, foi iniciado a implantação das normas técnicas do Sistema Brasileiro de Acreditação (SBA), coordenado pela Organização Nacional de Acreditação (ONA) (Brasil, 2014). Shaw et al. (2010), revela que os processos de acreditação em saúde iniciaram através da publicação dos Padrões Mínimos do Colégio Americano de Cirurgiões em 1917, que padronizava os processos para realização das cirurgias, melhorando a assistência hospitalar. A acreditação é uma metodologia de avaliação externa da qualidade, de caráter voluntário, com o intuito de averiguar conformidade dos padrões exigidos nos processos institucionais (Consórcio Brasileiro de Acreditação, 2010).

No cenário brasileiro, a regulamentação sanitária é gerenciada pela Agência Nacional de Vigilância Sanitária (ANVISA), que regulamenta registros de medicamentos, propagandas, boas práticas de funcionamento controlam vários tipos de estabelecimentos de saúde nas diversas complexidades, dentre outras atividades. Por sua vez a ANVISA na sua égide desempenhou competências para a proteção, amparo, defesa e elaboração das regulamentações que contribuam de maneira eficaz, controladora e estimuladora as práticas a segurança no paciente no Brasil através da RDC N. ${ }^{\circ} 45$ de 12 de Março de 2003, que dispõe sobre o regulamento Técnico de Boas Práticas de Utilização das Soluções Parenterais (SP) em Serviços de Saúde e que a inobservância ou desobediência a este disposto configura infração de natureza sanitária. Posteriormente, orientado pela OMS, o Ministério da Saúde (MS) no Brasil, sobre o controle da ANVISA instituiu através da Portaria MS no 529, de $1^{\circ}$ de Abril de 2013, o Programa Nacional de Segurança do Paciente, objetivando estratégias e a criação do Núcleo de Segurança do Paciente (NSP) em cada instituição de saúde, sendo responsável em pôr em prática as exigências ministeriais.

A segurança do paciente corresponde a promoção de ações positivas nas organizações da saúde, como: a redução e mitigação de atos não seguros, de erros não-intencionais, aplicação de boas práticas dos serviços de saúde, reduzindo os riscos e eventos adversos conforme o The Canadian Patient Safety Dictionary (Silva \& Rosa, 2016). A OMS conceitua a segurança do paciente como redução a um mínimo aceitável, o risco de dano desnecessário associado ao cuidado de saúde (Brasil, 2013; Brasil, 2013; Institute For Safe Medication Practices, 2018). Já o conceito da qualidade do cuidado em saúde e o alto nível de conhecimentos, aumento dos resultados positivos e redução dos resultados negativos (Brasil, 2003).

Em divergência as conformidades encontraram os eventos adversos evitáveis, que são erros que deveriam ser prevenidos e não foram, devido à falta de barreiras e processos adotados pela instituição. A OMS classifica estes erros como um Evento Adverso a Medicamentos (EAM) (Brasil, 2013). Estes incidentes devem ser notificados, conforme a Portaria $\mathrm{n}^{\circ}$ 1660, de 22 de Julho de 2009, que instituiu o Sistema de Notificação e Investigação em Vigilância Sanitária (Vigipos) do Sistema Único de Saúde (SUS). 
É normal na hospitalização, na terapia clínica, o uso de medicações sendo reconhecido como um dos pilares para regressão da enfermidade, contudo, o medicamento deve ser utilizado de forma coerente e racional, evitando-se erros e consequentemente a morbimortalidade dos pacientes (Rommers et al., 2007). Os profissionais de saúde além de seus conhecimentos adquiridos na formação devem ser atualizados e estimulados a continuidade dos estudos, evoluindo corriqueiramente com a evolução de novas tecnologias da saúde e a legislação sanitária brasileira.

Estes mesmos profissionais estão inclusos no Sistema de Medicação, sendo um processo que na maioria das vezes não tem o planejamento adequado por conta da dinâmica hospitalar e falta de diretrizes para normatização/acreditação, podendo ocorrer no seu percurso óbices, propiciando ao erro, perpetuando e desmembrando a sua a finalidade terapêutica e benéfica. É instituído de etapas compostas por: Aquisição, Recebimento, Armazenamento, Distribuição, Dispensação, Preparação, Administração e Investigação de Eventos adversos (Brasil, 2013), sendo antepenúltima e penúltima projetadas nesta pesquisa. Os erros que envolvem medicamentos são considerados incidentes evitáveis, podendo gerar danos aos pacientes e muitas vezes a causa da iatrogênia pode ser relacionada ao profissional, procedimentos, sistemas, falhas na prescrição, comunicação, etiquetação, envasamento, denominação, preparação, dispensação, distribuição, administração, educação, seguimento e utilização (Brasil, 2003).

Estes erros são piores quando envolvem os medicamentos de alta vigilância (MAV) que são conhecidos como de alto risco ou potencialmente perigosos, ou seja, possui maior potencial de causar danos graves ou até mesmo fatais, quando um erro é originado deste medicamento. Estudos mostram que as ocorrências com este tipo de medicamento são mais difíceis de acontecer, porém, quando acontecem as consequências ao usuário podem ser mais devastadoras (Institute For Safe Medication Practices, 2018). Uma pesquisa nos Estados Unidos no período de 2006 a 2008 estratificou do sistema americano MedMarx® o relatório sobre erros medicamentosos, apontando como aproximadamente 7\% dos 443.683 de erros relatados foram envolvendo MAV (Rashidee, 2009).

Segundo Cassiane et al. 2011, a unidade mais susceptível de erros com MAV é na unidade de terapia intensiva (UTI), acarretando comprometimentos mais graves, visto que o paciente já se encontra em deterioração clínica. O ISMP em 1995, realizou um levantamento histórico com 160 hospitais avaliando os medicamentos que desenvolveram danos graves e mortes, destacando-se a insulina, heparina, opióides, cloreto de potássio injetável, bloqueadores neuromusculares e quimioterápicos (Joint Commission Resources, 2008).

O Gerenciamento de Risco (GR) e o Núcleo de Segurança do Paciente (NSP) são indispensáveis nas instituições de saúde, visto que o internamento do paciente constitui uma imersão de periculosidade, sendo insubstituível a prevenção e a vigilância nas práticas de saúde. O eixo da farmacovigilância vem para potencializar as diretrizes e direcionamento para identificar, caracterizar, prevenir ou minimizar os riscos relacionados ao uso de medicamentos, incluindo a avaliação da efetividade (Brasil, 2013; Institute For Safe Medication Practices, 2018). A Joint Comission International (2010), recomenda a elaboração de políticas, protocolos e/ou procedimentos que orientam a localização, rotulagem e armazenamento de eletrólitos concentrados e de medicamentos de alta-vigilância (Silva, 2016).

O PNSP reforçou sobre os MAV, lançando boas práticas através do Protocolo de Segurança na Prescrição, Uso e Administração de Medicamentos em 2013 (Brasil, 2013), além do monitoramento de erros nas etapas do sistema de medicação e a criação de indicadores mensais. Destas boas práticas é de extrema importância ressaltar os cuidados em conjunto com os MAV: a dupla checagem (farmácia e enfermagem); dupla checagem para cálculos e administração; elaboração da listagem dos medicamentos da instituição com a forma de administração; limitação das apresentações e concentrações; suporte eletrônico para prescrição medicamentosa; restrição do número de apresentações e concentrações conforme padronização institucional; armazenamento com restrição de acesso, por ordem alfabética ou por formas farmacêuticas (identificadas com adesivo coloridos); identificação correta e diferente dos demais medicamentos armazenados; análise farmacêutica das prescrições; na 
dispensação, identificá-los; o farmacêutico tem que revisar as prescrições de MAV; permanência nos setores de internação os MAV que sejam extremamente necessários (Brasil, 2013; Institute For Safe Medication Practices, 2018).

Em 2017, houve uma conquista para Segurança do Paciente no Brasil, a publicação de regras para distinção gráfica e fonética em relação aos medicamentos e estabelecimento de critérios. Uma embalagem, nome, cor, etiqueta semelhantes pode causar confusão e propiciar o erro no preparo e administração do medicamento (Brasil, 2017). Entre os eventos evitáveis como este, é incluso também os relacionados às interações medicamentosas (IM), um erro silencioso, mas que também pode originar danos irreversíveis e até a morte. O erro envolvendo medicamento não necessariamente irá ser um evento adverso, porém, a vigilância ideal não deve ultrapassar nem a primeira barreira do incidente. O conceito do near miss ou do evento adverso em potencial, que é quando há um erro sem danos ao paciente, estes tipos de cenários é um campo ideal para o Gerenciamento de Risco mapear e avaliar o que está não conforme neste processo (Brasil, 2013; Frederico, 2012). Neste contexto, a farmacovigilância é o amparo científico e sanitário para o acompanhamento dos medicamentos comercializados, no intuito de identificar possíveis agravos à saúde dos usuários (Silva et al., 2014).

\section{Discussão}

Este estudo revelou que os profissionais de saúde que publicam sobre os medicamentos de alta vigilância/potencialmente perigosos, são enfermeiros e farmacêuticos, sendo ausente o papel de outros profissionais, como médicos, odontólogos, nutricionistas que também estão inseridos no sistema desta categoria de medicamento. Isto é um dado alarmante, visto que estes profissionais ausentes nos resultados são prescritores de medicamentos e de nutrição parenteral que deveriam fazer parte deste circuito.

A deficiência deste conhecimento, ou falta de interesse pode ser atribuído ao processo na formação universitária/técnica, e na falta de educação continuada no serviço. Sendo agravante por não ter posicionamento e conhecimento em caso de eventos adversos graves, tornando o paciente mais vulnerável ao erro (Engels \& Ciarkowski, 2015). O processo de acreditação hospitalar, hospital-escola, gerenciamento de risco, educação continuada e permanente, são alicerces para uma estimulação constante nas atividades profissionais e a capacitação dos mesmos, preenchendo as lacunas do conhecimento ou da descontinuidade da segurança e qualidade nos serviços.

Uma pesquisa recente empenhou-se em caracterizar o conhecimento sobre uso de MAV entre enfermeiros da assistência hospitalar, já que são responsáveis pelo processo de preparo e administração dos medicamentos e da supervisão do trabalho de técnicos de enfermagem, como resultado foi obtido um nível não satisfatório sobre as técnicas e conhecimentos prévios daqueles medicamentos que estavam sendo prescritos. Estudos mostram que mais de $90 \%$ dos erros com MAV concentram-se nos medicamentos: heparina, fentanil, midazolam, nalbufina, pancurônio, dopamina, cloreto de potássio, tramadol, epinefrina, petidina e morfina, sendo relacionados a prescrição medicamentosa não conforme, gerando erros de prescrição, preparo e administração (Santos et al., 2020).

Os anestésicos e os eletrólitos também foram citados com uma percentagem significativa, respectivamente, 10,7\% e 5,33\% (Rosa et al., 2009). As interações medicamentosas também é outro problema quando não se tem conhecimento farmacológico, foram encontradas numa pesquisa com 244 prescrições individuais de pacientes, 846 interações medicamentosas potenciais relacionadas aos MAVs. Na maioria destes erros envolviam midazolam, fentanil ou insulina regular, esta pesquisa foi realizada numa unidade de terapia intensiva, nas quais os pacientes estavam em estado crítico, ou seja, devido ao quadro ruim do paciente, há uma necessidade maior no uso destes medicamentos e durante uma deterioração clínica pode ser prescritos por ordem verbal devido a emergência do caso, e/ou rapidez no preparo, facilitando assim desta forma as iatrogênias, podendo ser um dos fatores multicausais para o erro (Cortes et al, 2019). A busca resultou num artigo que 
mencionava mortes associadas aos MAVs, destacando os opióides, benzodiazepínicos, anticoagulantes e insulina (Smeulers et al., 2015).

Não temos no Brasil, dados expressivos e fidedignos sobre todos os eventos adversos que ocorrem, eventos sentinelas, os óbitos oriundos ao erro e de sequelas permanentes ou temporárias. A ANVISA implantou em 2019, o sistema VigiMed, estimulando a notificações de eventos adversos provocados por medicamentos no território brasileiro, facilitando o acesso e os tratamentos dos casos. Desta forma, é detectável o aumento de notificações, em 2019, a ANVISA recebeu 2.771 notificações (Brasil, 2020). Atualmente no país, de acordo com a ANVISA, são 4.612 Núcleos de Segurança do Paciente, sendo um número ainda incipiente, visto o vasto crescimento de estabelecimentos de saúde e a permanência sem núcleos.

A cultura de segurança no Brasil progride de acordo com a percepção dos profissionais de saúde sobre qualidade e relação aos processos de acreditação, existe uma estratégia ineficaz nos processos assistenciais da saúde, existe uma formação acadêmica com lacunas, na qual não inclui a segurança do paciente na sua grade curricular. Não é ponto de discussão o estreitamento na relação entre a gestão da qualidade em saúde e a educação permanente para sensibilização e treinamento do pessoal conforme é previsto em portaria ministerial (Institute For Safe Medication Practices, 2018). É necessário que haja uma mudança na cultura organizacional e a valorização da qualidade em saúde, da mesma forma que é exaltado um erro, uma iatrogênia.

Conforme a WHO (2017), os erros decorrentes da terapia medicamentosa são resultados de processos falhos e procedimentos durante o cuidado. É susceptível em qualquer etapa do sistema de medicação: prescrição, dispensação, preparação, administração e monitoramento, sendo responsabilidade de todos os profissionais, o prescritor, o dispensador, farmacêutico, o que prepara e administra profissionais da enfermagem e o monitoramento que é função de todos.

\section{Conclusão}

A realidade brasileira é delicada quando envolve um controle fidedigno sobre os medicamentos de alta vigilância, os dados existentes são inexpressivos, sendo incipientes, demonstrando a realidade de um estabelecimento de saúde ou município, não tratando a realidade da abrangência do país e/ou por regionalidade. Os eventos adversos evitáveis e os fatores contribuintes podem ser modificados através de tecnologias leves e treinamentos sem grandes custos. Há necessidade de investimento na educação continuada nos estabelecimentos de saúde e de ser introduzido na formação acadêmica e técnica de saúde.

Mesmo com o empenho de campanhas internacionais como da OMS em 2017, com o $3^{\circ}$ Desafio Global, onde pretende reduzir 50\% dos erros de medicação até 2022, percebe-se que o Brasil ainda não tem números reais para iniciar a estratégia de alcance desta meta. Há diferenças entre regiões do país, escassez de profissionais em algumas delas, hospitais privados, filantrópicos e públicos, incentivos com programas como a Rede Sentinela, mesmo diante de um cenário heterogêneo, cada unidade deve buscar as premissas básicas que são propostas na PNSP.

Desde 2013, que está incluso na PNSP a inclusão nas grades curriculares de ensino a saúde a propagação do conteúdo em segurança do paciente, e muitos profissionais, nunca ouviram falar em conceitos básicos e/ou indicadores. Em contrapartida, a maioria das instituições não preenche estas lacunas dos seus servidores, não existe qualidade sem processos eficazes na conjuntura atual. Uma das recomendações é a elaboração de protocolos assistenciais, checklist, ferramentas que acompanhe e direcione este profissional para um processo seguro e eficaz. Ficou evidente a escassez de artigos nos últimos anos sobre o papel e cuidados dos prescritores de medicações.

São recomendadas ações que promovam a segurança desde o pedido da compra do medicamento pelo setor responsável, dando prioridades às indústrias que respeitam a legislação e a rotulagem que promova um ambiente seguro. É conclusivo que ao final deste levantamento, o problema é multicausal e têm vários óbices, um sistema com vários acessos, 
como é o da medicação, e tratando-se de vários profissionais de nível superior e médio incluso, a falta de supervisão ou fiscalização propicia aos erros.

Por tratar-se de uma temática tão relevante na saúde pública mundial, visto a potencialidade de erros com os medicamentos de alta vigilância e seus eventos adversos, fica a sugestão para ampliação de pesquisas na área, podendo ser especificando cada medicamento ou por complexidade hospitalar e ambulatorial. A segurança do paciente e principalmente o uso correto dos medicamentos deve ser reverberada desde formação profissional até a educação continuada de forma paulatina e perene aos profissionais que estão na ponta da assistência, garantindo uma prática segura em saúde.

\section{Agradecimentos}

À Coordenação de Aperfeiçoamento de Pessoal de Nível Superior pelo apoio financeiro para o desenvolvimento da ciência. Ao Programa de Pós Graduação em Inovação Terapêutica pelo fomento à realização e produção de novos trabalhos na área acadêmica.

\section{Referências}

Brasil. (2013). Agência Nacional de Vigilância Sanitária - ANVISA. Resolução da Diretoria Colegiada da Anvisa - RDC nº 36 , de 25 de julho de 2013. https://bvsms.saude.gov.br/bvs/saudelegis/anvisa/2013/rdc0036_25_07_2013.html

Romero, M. P., González, R. B., Calvo, M. S. R., \& Fachado, A. A. (2018). A segurança do paciente, qualidade do atendimento e ética dos sistemas de saúde. Rev. Bioét. vol.26 no.3 Brasília. https:/www.scielo.br/j/bioet/a/4hRnkzkJFL8MxdRByNv7LPj/?format=pdf\&lang=pt

Brasil. (2014). Implantação do Núcleo de Segurança do Paciente em Serviços de Saúde - Série Segurança do Paciente e Qualidade em Serviços de Saúde. https://segurancadopaciente.com.br/wp-content/uploads/2015/09/ebook-anvisa-06-implantacao-do-nucleo-de-seguranca-do-paciente-em-servicos-de-saude.pdf

Brasil. (2013). Assistência Segura: Uma reflexão teórica aplicada à prática. Série Segurança do Paciente e Qualidade nos serviços de saúde. 1. http://www.saude.pi.gov.br/uploads/divisa_document/file/374/Caderno_1_-_Assist\%C3\%AAncia_Segura_-

_Uma_Reflex\%C3\%A3o_Te\%C3\%B3rica_Aplicada_\%C3\%A0_Pr\%C3\%

Sheikh, A., Dhingra-Kumar, N., Kelley, E., Kieny, P., \& Donaldson, L. J. (2017). The third global patient safety challenge :tackling medication-relatedharm. Bull World Health Organ. 95:546-546A. https://pubmed.ncbi.nlm.nih.gov/28804162/

Miasso, A. I., Silva, A. E. B. C., Cassiani, S. H. B., Grou, C. R., \& Fakih, F. T. (2006). Erros de medicação: tipos, fatores causais e providências tomadas em quatro hospitais brasileiros. RevEscEnferm USP. 2006;40(4):524-32. https://www.scielo.br/j/reeusp/a/D8nKpL3rZYrB4KhpPnmL78L/?lang=pt

Curran, C. R., \& Totten, M. K. (2011). Governing for improved quality and patient safety. Nursing Economics. 29(1):38-41. http://nursingeconomics.net/necfiles/BestonBoard/JF_11_BoB.pdf

World Health Organization. (2009). Taxonomy. The Conceptual Framework for the International Classification for Patient Safety. https://www.who.int/patientsafety/taxonomy/icps_full_report.pdf

Brasil. (2013). Protocolo de segurança na prescrição, uso e Administração de medicamentos. https://repositorio.observatoriodocuidado.org/bitstream/handle/handle/1650/protoc_identificacaoPaciente.pdf?sequence=1\&isAllowed=y

Institute For Safe Medication Practices. (2018). High-Alert Medication Survey Results Lead to Several Changes for 2018. https://www.ismp.org/resources/high-alert-medication-survey-results-lead-several-changes-2018

Echer, I.C. (2001). A revisão de literatura na construção do trabalho científico. Revista gaúcha de enfermagem. https://lume.ufrgs.br/handle/10183/23470

Duarte, S. C. M., Stipp, M. A. C., Silva, M. M., \& Oliveira, F. T. (2015). Eventos adversos e segurança na assistência de enfermagem. Rev Bras Enferm. https://www.scielo.br/j/reben/a/mBxyRmzXxjVYbDQZfg7phyj/?format=pdf\&lang=pt

Kohn, L. T., Corrigan, J. M., \& Donaldson, M. S. (2001). To Error is human: building a safer health system: a report of the Committee on Quality of Health Care in America.

Brasil. (2014). Manual das Organizações Prestadoras de Serviços de Saúde. Brasília: ONA.

Shaw, C., Groene, O., Moura, N., \& Sunol, R. (2010). Accreditation and ISO certification: do they explain differences in quality management in European hospitals? International Journal for Quality in Health Care. https://pubmed.ncbi.nlm.nih.gov/20935006/

Consórcio Brasileiro De Acreditação. (2010). Padrões de acreditação da Joint Commission Internacional para hospitais.

Brasil. (2003). RDC n. 45, de 12 de março de 2003. https://bvsms.saude.gov.br/bvs/saudelegis/anvisa/2003/rdc0045_12_03_2003.html

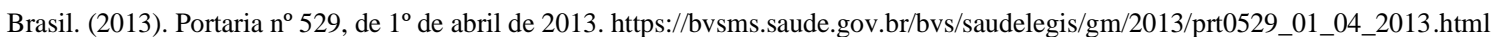


Research, Society and Development, v. 11, n. 2, e4511225491, 2022

(CC BY 4.0) | ISSN 2525-3409 | DOI: http://dx.doi.org/10.33448/rsd-v11i2.25491

Silva, A. C. A. B., \& Rosa, D. O. S. (2016). Cultura de segurança do paciente em organização hospitalar. Cogitare Enferm. https://revistas.ufpr.br/cogitare/article/view/45583

Rommers, M. K., Teeepe-Twiss, I. M., Guchelaar, H. J. (2007). Preventing adverse drugevents in hospital practice: an overview. Pharmacoepidemiol DrugSaf. https://pubmed.ncbi.nlm.nih.gov/17610221/

Rashidee, A., Hart, J., Chen, J., \& Kumar, S. (2009). High-Alert Medications: Error Prevalence and Severity. Patient Safety \& Quality Healthcare.

Cassiani, S. H. B., Deus, N. N., \& Capucho, H. C. (2011). Administración segura de medicamentos.

Joint Commission Resources. (2008). High-Alert Medications: Strategies for Improving Safety.

Brasil. (2017). Boletim Segurança do Paciente e Qualidade em Serviços de Saúde $\mathrm{n}^{\circ}$ 15: Incidentes Relacionados à Assistência à Saúde. https://www.gov.br/anvisa/pt-br/centraisdeconteudo/publicacoes/servicosdesaude/boletim-seguranca-do-paciente/boletim-seguranca-do-paciente-e-qualidadeem-servicos-de-saude-no-15.pdf

Frederico, P. M. (2012). Interações medicamentosas potenciais dos antihipertensivos: uso perigoso entre idosos. Fundação Oswaldo Cruz, Escola Nacional de Saúde Pública. https://www.arca.fiocruz.br/handle/icict/24287

Silva, P. L., Cornélio, R. A. C., \& Araújo, A. L. A. (2014). Farmacovigilância: conhecimento e ação dos profissionais frente a desvios de qualidade de medicamentos. Rev Bras Farm Hosp Serv Saúde. http://rbfhss.saude.ws/revista/arquivos/2014050107000475BR.pdf

Engels, M., \& Ciarkowski, S. (2015). Nursing, pharmacy, and prescriber knowledge and perceptions of high-alertmedications in a large, academic medical hospital. Hosp Pharm. https://www.ncbi.nlm.nih.gov/pmc/articles/PMC4585568/

Santos, G. O., Farre, A. G. M. C., Santana, I. T. S., Rocha, H. M. N., Carvalho, A. A., Santos, G. K. B. B. et al. (2020). Knowledge about the use of potentially dangerous drugs among hospital health care nurses. Rev Rene. http://periodicos.ufc.br/rene/article/view/44466

Rosa, M. B., Perini, E., Anacleto, T. A., Neiva, H. M.; \& Bogutchi, T. (2009). Erros na prescrição hospitalar de medicamentos potencialmente perigosos. Rev Saúde Pública. https://www.scielo.br/j/rsp/a/XwLXkGKCS9g5wTTNtL6nXSh/abstract/?lang=pt\#: :text=RESULTADOS\%3 A\%20Houve\%20 predo m\%C3\%ADnio\%20da\%20prescri\%C3\%A7\%C3\%A3o,estavam\%20pouco\%20leg\%C3\%ADveis\%20ou\%20ileg\%C3\%ADveis.

Cortes, A. L. B., Silvino, Z. R., Santos, F. B. M., Pereira, J. A. C., \& Tavares, G. S. (2019). Prevalência de interações medicamentosas envolvendo medicamentos de alta-vigilância: estudo transversal. REME - Rev Min Enferm. http://reme.org.br/artigo/detalhes/1372

Smeulers, M., Verweij, L., Maaskant, J. M., De Boer, M., Krediet, C. T. P., Nieveen Van Dijkum, E. J. et al. (2015). Quality indicators for safe medication preparation and administration: a systematic review. PLoSOne. https://pubmed.ncbi.nlm.nih.gov/25884623/

Brasil. (2020). Boletim de Farmacovigilância aborda erros de medicação. http://antigo.anvisa.gov.br/resultado-debusca?p_p_id=101\&p_p_lifecycle=0\&p_p_state=maximized\&p_p_mode $=$ view\&p_p_col_id $=$ column-

1\&p_p_col_count=1\&_101_struts_action=\%2Fasset_publisher\%2Fview_content\&_101_assetEntryId=5765434\&_101_type=content\&_101_groupId=219201 \&_101_urlTitle=boletim-de-farmacovigilancia-aborda-erros-de-

medicacao\&inheritRedirect=true\#: :text=A1\%C3\%A9m\%20de \%20n\%C3\%A3o\%20atender\%20adequadamente,a\%20hospitaliza\%C3\%A7\%C3\%B5es\%20e

$\% 20$ causar\%20mortes.\&text=Os\%20erros\%20de\%20medica\%C3\%A7\%C3\%A3o\%20representam,precisam\%20ser\%20relatados\%20e\%20monitorados.

World Health Organization. (2017). Medicantion withoutharm: WHO global patient safety challenge. https://www.who.int/initiatives/medication-withoutharm 